\title{
High Throughput Multimodal Neuroimaging for Alzheimer's Disease (AD)
}

\author{
Shiza Fatima ${ }^{1}$, Mohsin Jamil ${ }^{2}$ and Syed Omer Gilani ${ }^{1}$ \\ ${ }^{1}$ Department of Biomedical Engineering and Sciences \\ ${ }^{2}$ Department of Robotics and Artificial Intelligence \\ School of Mechanical and Manufacture Engineering (SMME), \\ National University of Sciences and Technology (NUST), \\ Islamabad Pakistan \\ shizzafatimasyed@gmail.com,mohsin@smme.nust.edu.pk, \\ omer@smme.nust.edu.pk
}

\begin{abstract}
Alzheimer's disease (AD) is a neurodegenerative disorder characterized by the development of plaques and neurofibrillary tangles in nerve cells leading to their death, and dementia. With advancements in medical imaging processing, over the past few decades, neuro-imaging has surfaced as an essential component in assessment and diagnosis of neural disorders. Integration of correlation analysis, between control and $A D$ 's and MRI dataset, can be used to develop a reference, to which new patient's data can be compared for initial diagnosis of $A D$. By implementing proposed framework, it is possible to acquire, simultaneously, enhanced images and dataset for comparing data of existing $A D$ patients and incoming/new patients. Thus, we propose to develop a reference dataset against which other images can compared.
\end{abstract}

Keywords: Alzheimer's disease (AD, MRI, noise reduction, image enhancement

\section{Introduction}

Alzheimer is a neurodegenerative disorder characterized by progressive death of brain cells over a period of time [1]. Alzheimer is primarily identified by decline in cognitive abilities. Emphasis is being laid on neurotransmitter release, whose altered concentration might be involved in symptomatic manifestation of Alzheimer (2). Conclusive evidence suggests role of cholinergic neurons i.e., acetylcholine releasing neurons, residing in basal forebrain, undergo selective degeneration (3). Cerebral cortex has innervations from cholinergic neurons and play an essential part in cognitive functions, foremost of which is memory (4). Cell death leads to loss of cognitive abilities and memory. The underlying cause of Alzheimer's disorder is attributed plaques and tangle on nerve cells, as indicated during autopsy [7]. Accumulation of beta-amyloid protein between dying cells forms plaques while degeneration of tau protein within neurons form tangles. Beta-amyloid protein accumulation drive Alzheimer pathogenesis (5). Neurofibrillary tangles containing tau protein lead to imbalance between $A \beta$ production and $A \beta$ clearance (6). It effects people over the age of 65 and results in diminished cognitive abilities. Type II-diabetes, hypertension, obesity, smoking and dyslipidemia act as risk factors for Alzheimer's. Build-up of abnormal clumps and inclusions might not be the only cause of Alzheimer's, scientists are probing into other underlying reasons [2]. Higher concentration of aluminum is associated enhanced induction of neurofibrillary degeneration (7). 
Estrogen, a primary female hormone responsible for development and regulation of female reproductive system, has effect on brain of both male and female (8). It has profound effect on neuronal synapse formation in hippocampus with evidence suggesting different response of brain towards hormone. Estrogen withdrawal in female due to menopause or hysterectomy might act as a risk factor for Alzheimer (9).

Modern neuroimaging data, positron emission tomography and functional magnetic resonance imaging (fMRI), has unveiled information present in the deep and convoluting folds of insula gyri (10). Current bioinformatic tools, with improved performance, have made early diagnosis of Alzheimer possible. Improved Alzheimer disease classification images have been obtained via hybrid MRI imaging (11).

Image processing and analyses tools have rendered clinicians and radiologist with diagnose and treat myriad diseases. Over the past few decades, advancements in image processing techniques have played a vital role in diagnosis and treatment of Alzheimer's [3].

As for its diagnosis, to date, biomarkers are available which prevent and slow down AD's progression but not efficient enough to predict $100 \%$ diagnosis. Various approaches like Distributed spectral embedding (DSE) [5], Multi-modal spectral embedding method (SMSE) [4], and Multi-view spectral embedding algorithms have been put forth as effective treatment modality and for accurate diagnosis. Akhila DB et al., [7] proposed a framework for diagnosis of AD from multimodal neuroimaging features. They reported findings on the PET and MRI datasets which are publicly available under ANDI parent database. They based their research on data fusion which rendered high throughput diagnosis.

Integration of correlation analysis, between control and AD's and MRI dataset, can be used to develop a reference, to which new patient's data can be compared for initial diagnosis of $\mathrm{AD}$. By implementing proposed framework, it is possible to acquire, simultaneously, enhanced images and dataset for comparing data of AD patients and incoming patients i.e., to develop a reference against which other datasets may be compared.

\section{Methodology}

\subsection{Input dataset-MRI data}

Neuroimaging data is obtained from OASIS (Open Access Series of Imaging Studies). It is an open source database, making neuroimages freely available to scientific community. Twenty cross sectional Magnetic Resonance Imaging (MRI) scans of Alzheimer's patients are accessed along with equal number of scans of normal individuals acting as control group. To see the progression of Alzheimer's disorder, MRI based images are used as input modality. To fuse different MRI image we use affine transform technique as it is robust and efficient way to align images.

\subsection{Pre-processing}

Pre-processing improves the quality of image rendering it more reliable for proceeding phases. Weiner filter, a noise removal filter, is used to eliminate additive noise from MRI scans while preserving important details of the image. This filter reduces the noise component by comparing it desired noiseless image. Reduction of Mean Square Error (MSE) is the main objective of Weiner filter. Mean square error is a quantitative measure computed over pixel intensity level between raw and noisy image.

\subsection{Image Enhancement}

To improve feature detection and structural information in images for automated analysis, image enhancement is a powerful technique. MRI scans are enhanced by 
histogram equalization technique in the proposed framework. It improves contrast in the image. The output of this technique is improved and enhanced image with a uniform histogram.

\subsection{Region of Interest based Processing}

Portion of an image on which myriad operations can be performed is region of interest (ROI). ROI is defined by creating a binary mask which renders value 1 to pixels demarcating ROI while remaining values become 0 . Region of interest filtering is applied on correlated images as it involves filter application on a distinct part of image, that region being defined by binary mask. Thus, improving intensity of an image.

In order to perform ROI based operation following are the pre-requisites:

a) Greyscale input image

b) Binary mask image that defines ROI

c) A filter

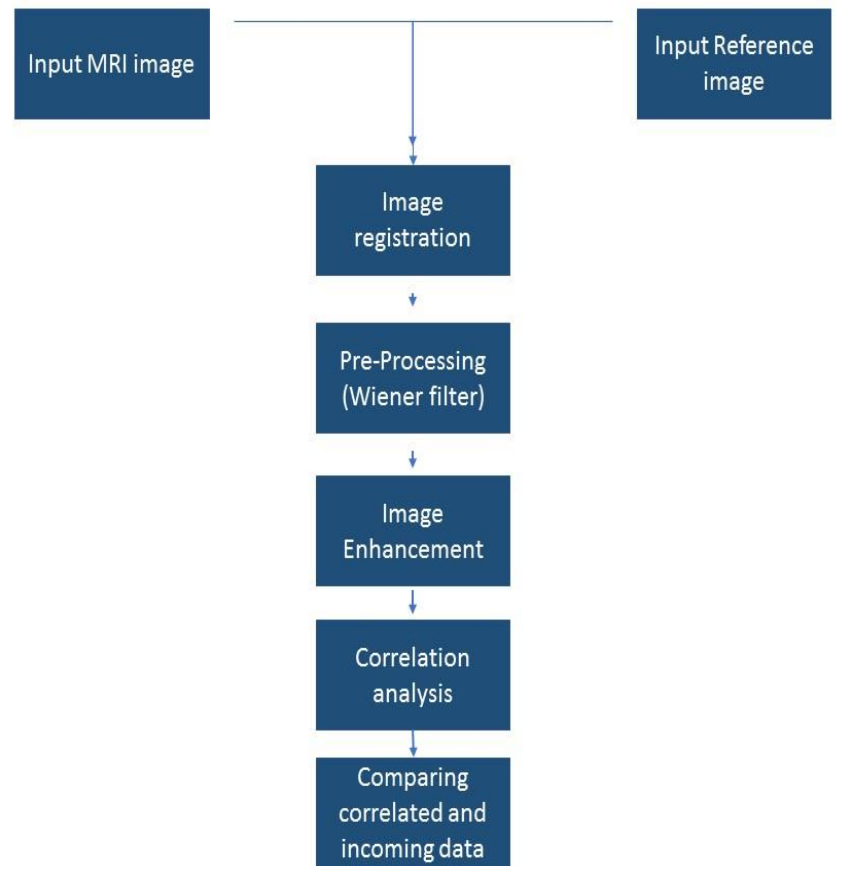

Figure 1. Proposed Methodology

\section{Results}

MRI scans and scans of individuals without Alzheimer's i.e., of normal subjects were used for image analysis. Both types of images were of 8 bits with $256 \times 256$ dimensions. Image registration was carried out by using imregister command. Unregistered and registered images are shown in Figure $1(a, b)$. Matlab 2013 was used for development of proposed algorithm. 

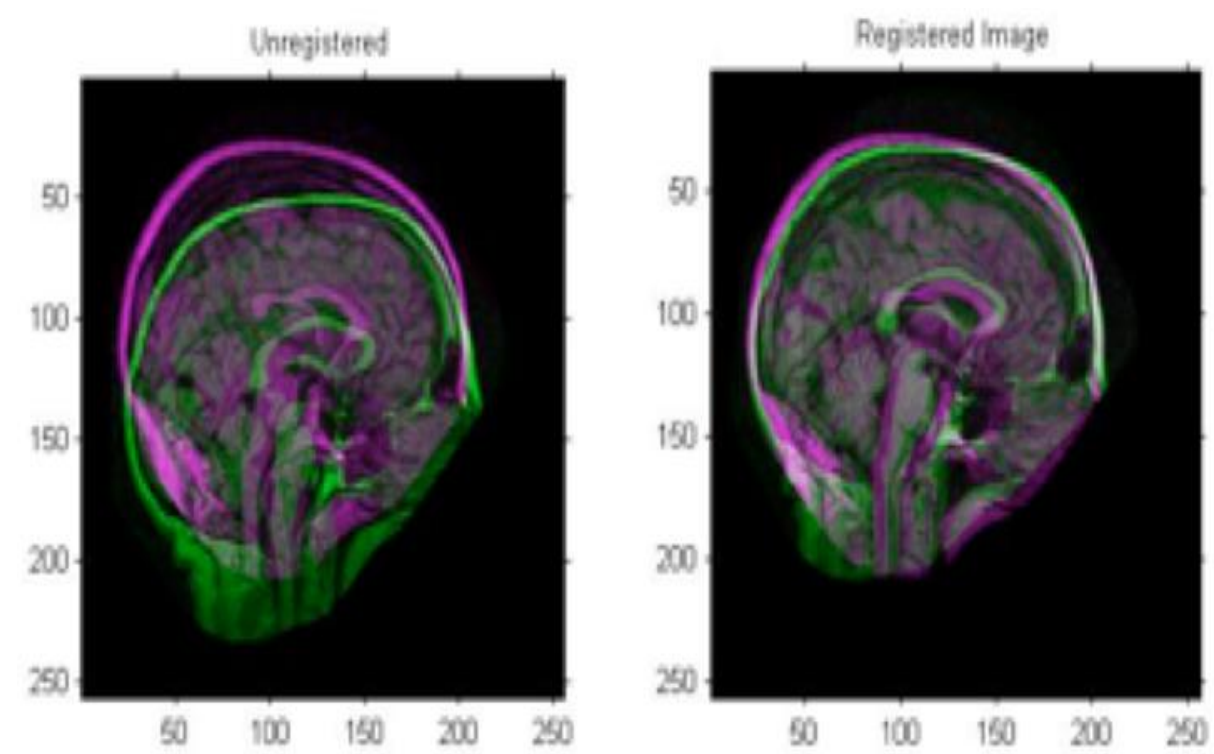

Figure 2. Image registration (left) Unregistered Image (Right) Registered Image

Pre-processed MRI scan by Wiener filter is shown in Figure3(a). Application of filter results in blurred image. Due to blurring, image was enhanced using histogram equalization. Results of equalization are shown in Figure3(b).
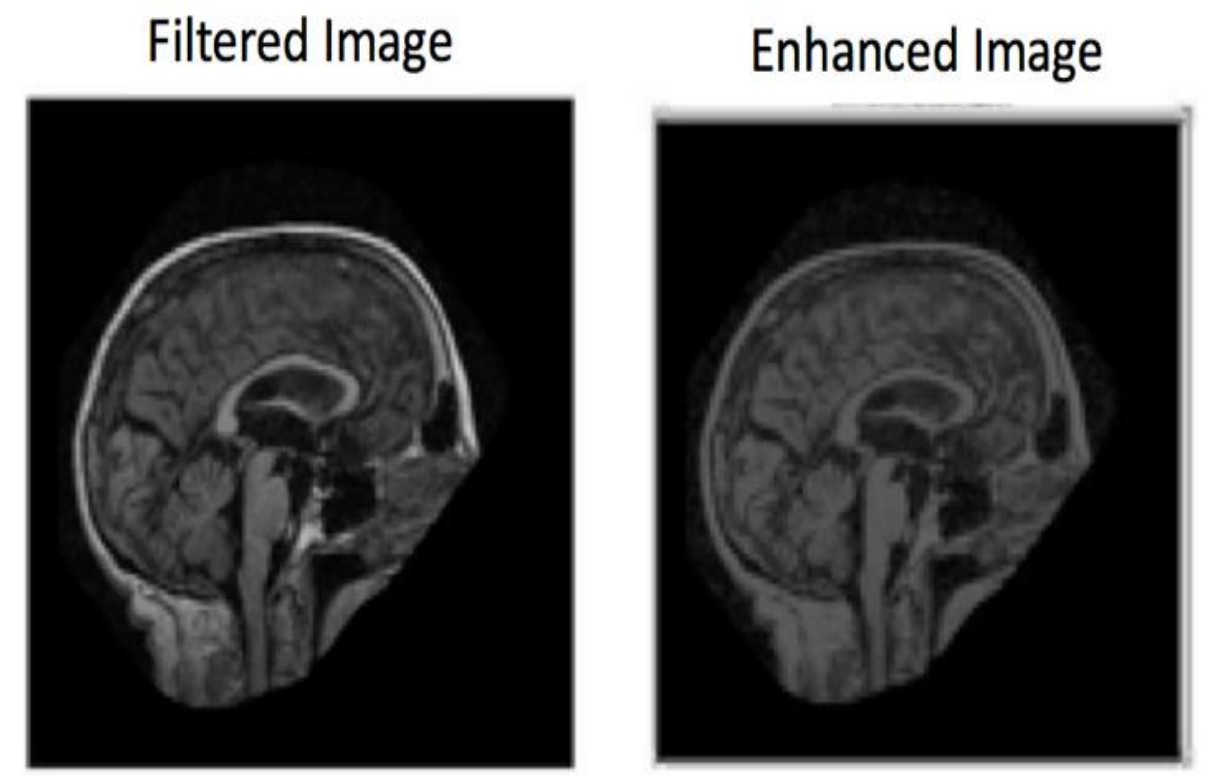

Figure 3. (A) Image Filtering by Wiener Filter (right) Image Enhacement by Histogram Equalization

Edge detection, using Canny filter, was used to highlight structural information in the brain (Figure 4). 


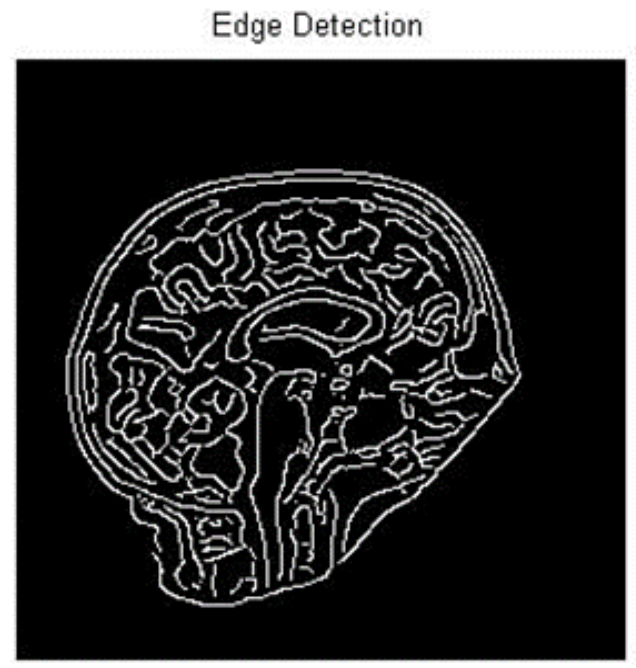

Figure 4. Edge Detection using Canny Filter

Image was further sharpened and its contrast was adjusted for follow-up registration process (Figure 5).

\section{Sharpened Image}

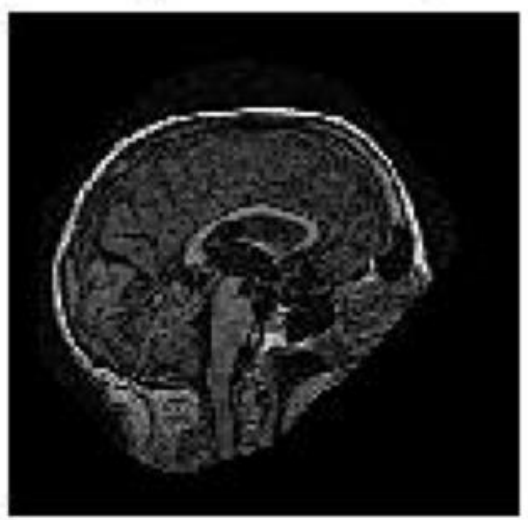

\section{Contrast adjusted Image}

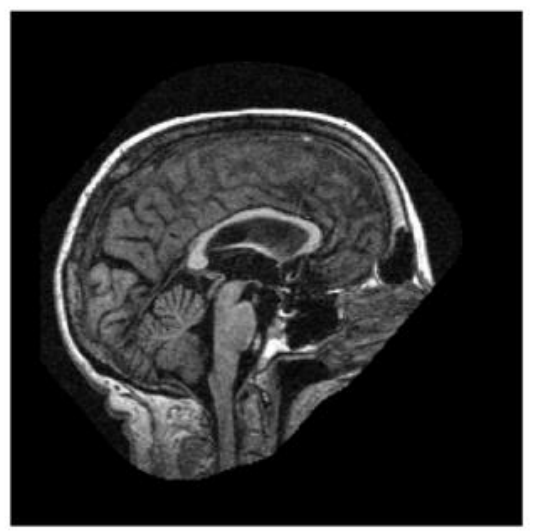

Figure 5. (left) Sharpened Image (right) Contrast Adjusted Image

Finally, we show results of registration in Figure 6(a), using similarity score model. The similarity score is computed using point-wise correlation between image pixel values. A zoomed-in view of different anatomical structure of the brain is shown in Figure 6 (b) along with corresponding similarity score in Figure6(c). A higher score (labeled using white) indicates that boundaries of these brain structure have properly aligned during registration process.

Distinct areas of an image or region of interest (ROI) can be processed for in depth analysis using region of interest (ROI) based processing. Region of interest is a part of image selected for performing filters or some other operations. A binary mask defines the region of interest. In mask pixel values for ROI are 1 while all other values are set to 0. Roipoly syntax specifies a polygonal region of interest. It returns a binary image that is used as a ma 
Region of interest can be enhanced by intensity adjustment operation via ROI filtering. The process involves filtering region of interest using binary mask. roifilt 2 command filters the input image and returns the filtered image.
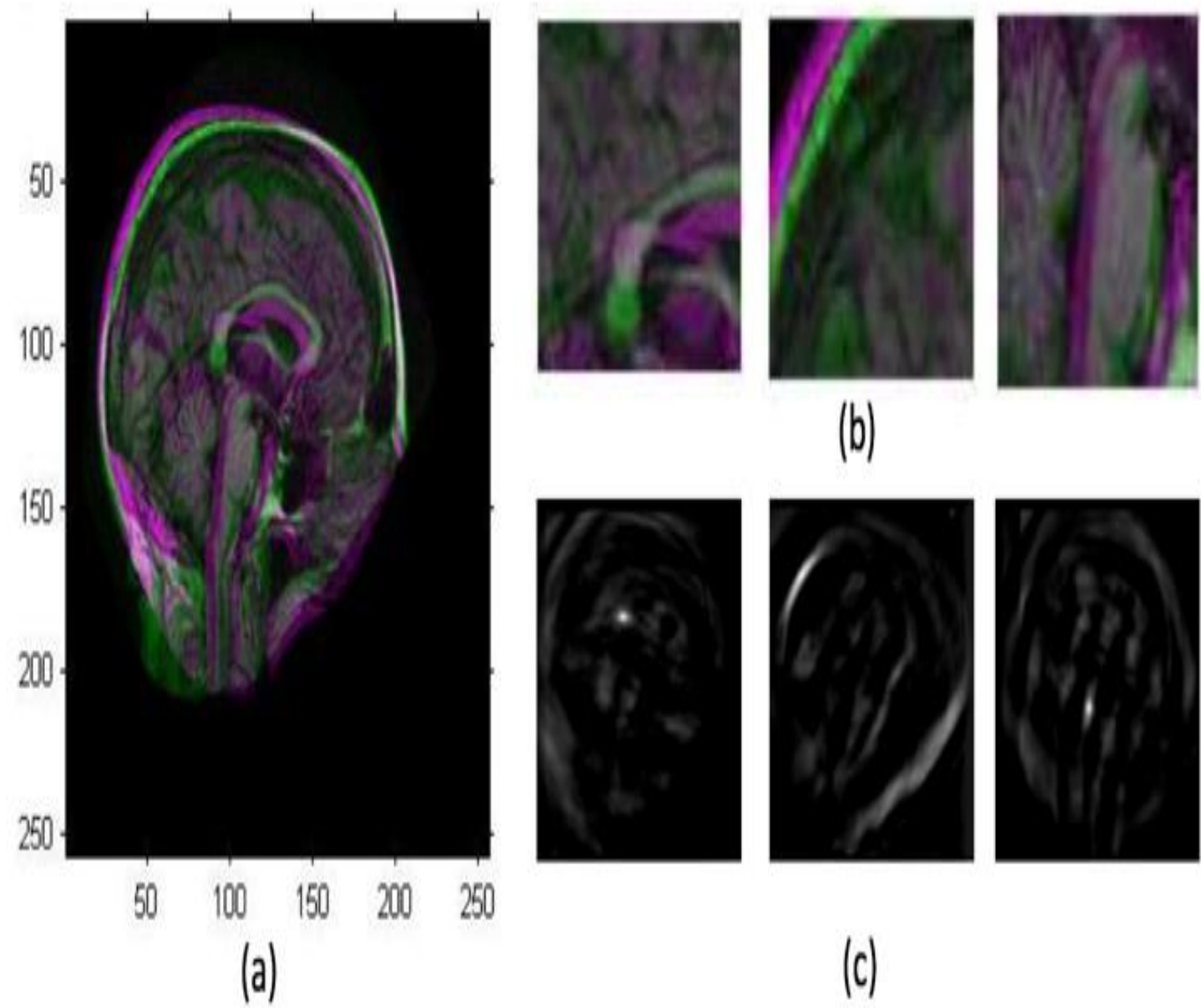

(b)
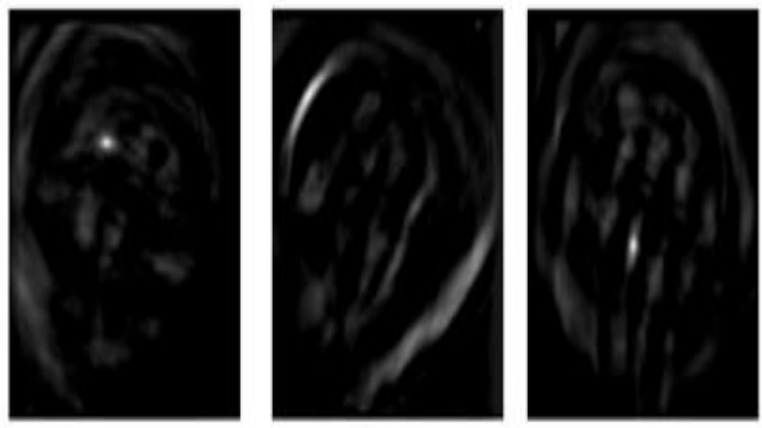

(a)

(c)

Figure 6. (a) Image Registration using Similarity Score Model. (b) A Zoomed-In View of Different Brain Areas Frontal, Parietal, and Temporal (from Left to Right). (c) Corresponding Similarity Score for each Area

\section{Conclusion}

Image registration, used for image alignment, extracted information that would otherwise be impossible to obtain from single image. It also improved signal to noise ratio. Target image (moving) was aligned to reference (fixed) image. Intensity based registration was used to align images according to their relative intensity pattern. Edge detection was used to demarcate specific brain areas. Other enhancement techniques were used for better image presentation and analysis. Since, Alzheimer's usually affects frontal, parietal, and temporal lobes; correlation was used to locate these areas. Correlation analysis, between control and AD's MRI dataset, can be used to develop a reference, to which new patient's data can be compared for initial diagnosis of AD. The aforementioned lobes of brain can be further processed for in depth analysis using region of interest (ROI) filter. Once correlation is performed, area specific enhancement can render better results in terms of image quality. By implementing proposed framework, it is possible to acquire, simultaneously, enhanced images and dataset for comparing data of $\mathrm{AD}$ patients and incoming patients i.e., to develop a reference against which other datasets may be compared. 


\section{References}

[1] G. McKhann, D. Drachman, M. Folstein, R. Katzman, D. Price and E. M. Stadlan, "Clinical diagnosis of Alzheimer's disease: report of the NINCDS-ADRDA Work Group under the auspices of Department of Health and Human Services Task Force on Alzheimer's Disease", Neurology, vol. 34, no. 7, (1984), pp. 939-44.

[2] R. T. Bartus, Rr Dean, B. Beer and A. S. Lippa, "The cholinergic hypothesis of geriatric memory dysfunction”, Science, vol. 217, no. 4558, (1982), pp. 408-14.

[3] P. J. Whitehouse, D. L. Price, R. G. Struble, A. W. Clark, J. T. Coyle and M. R. Delon, "Alzheimer's disease and senile dementia: loss of neurons in the basal forebrain", Science, vol. 215, no. 4537, (1982), pp. 1237-9.

[4] J. Coyle, D. Price and M. DeLong, "Alzheimer's disease: a disorder of cortical cholinergic innervation", Science, vol. 219, no. 4589, (1983), pp. 1184-90.

[5] J. Hardy and D. J. Selkoe, "The Amyloid Hypothesis of Alzheimer's Disease: Progress and Problems on the Road to Therapeutics", Science, vol. 297, no. 5580, (2002), pp. 353-6.

[6] J. A. Hardy and G. A. Higgins, "Alzheimer's disease: the amyloid cascade hypothesis", Science, vol. 256, no. 5054, (1992), pp. 184

[7] D. R. Crapper, S. S. Krishnan and A. J. Dalton, "Brain Aluminum Distribution in Alzheimer's Disease and Experimental Neurofibrillary Degeneration", Science, vol. 180, no. 4085, (1973), pp. 511-3.

[8] S. J. Lupien, B. S. McEwen, M. R. Gunnar and C. Heim, "Effects of stress throughout the lifespan on the brain, behaviour and cognition", Nature reviews Neuroscience, vol. 10, no. 6, (2009), pp. 434.

[9] G. Einstein, A. Au, D. Schwartz, E. Hampson, M. C. Tierney and W. Meschino, "Sex Differences in the Brain and Implications for Alzheimer\&\#X2019;S Disease", Alzheimer's \& Dementia: The Journal of the Alzheimer's Association, vol. 12, no. 7, pp. 161.

[10] I. D. Kilsdonk, M. P. Wattjes and J. J. Geurts, "Ultra-high field MRI: looking through the 'macroscope", Journal of Neurology, Neurosurgery \&amp; Psychiatry, vol. 85, no. 1, (2014).

[11] D. Chen, A. Alsadoon, P.W. C. Prasad and A. Elchouemi, "Early diagnosis of Alzheimer using mini mental state examination method", MMSE. 2017 8th International Conference on Information and Communication Systems (ICICS); (2017) April 4-6.

[12] R. Brookmeyer, E. Johnson, K. Ziegler-Graham and H. M. Arrighi, "Forecasting the global burden of Alzheimer's disease", Alzheimer's \& dementia: the journal of the Alzheimer's Association, vol. 3, no. 3, (2007), pp. 186-91.

[13] E. Cavedo, S. Lista, Z. Khachaturian, P. Aisen, P. Amouyel and K. Herholz, "The Road Ahead to Cure Alzheimer's Disease: Development of Biological Markers and Neuroimaging Methods for Prevention Trials Across all Stages and Target Populations", The journal of prevention of Alzheimer's disease, vol. 1, no. 3, (2014), pp. 181-202.

[14] S. Liu, L. Zhang, W. Cai, Y. Song, Z. Wang and L. Wen, "A supervised multiview spectral embedding method for neuroimaging classification", 2013 IEEE International Conference on Image Processing; 2013 (2013) September 15-18.

[15] S. Liu, W. Cai, L. Wen and D. Feng, "Multi-Channel brain atrophy pattern analysis in neuroimaging retrieval”, 2013 IEEE 10th International Symposium on Biomedical Imaging; (2013) April 7-11.

\section{Authors}

Shiza Fatima is currently Masters Student at Department of Biomedical Engineering and Sciences within NUST School of Mechanical and Manufacturing Engineering (SMME). Her research interests include biomedical imaging.

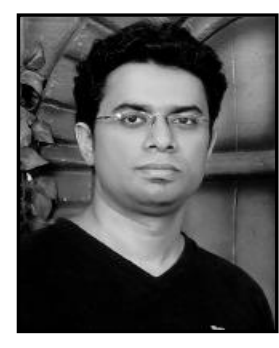

Syed Omer Gilani, Syed Omer Gilani is currently an assistant professor at National University of Sciences and Technology (NUST), Pakistan. He earned his Ph.D. in electrical and computer engineering from National University of Singapore in 2013 and MSc degree in computer engineering from Sweden in 2006. Between 2006 and 2008, he worked at Interactive Multimedia Lab (Singapore). His research interests include human-machine interaction and networking and actively consults for industry on various projects. 


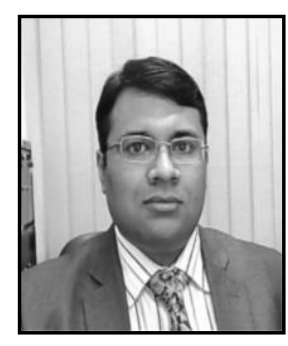

Mohsin Jamil, Mohsin Jamil is currently assistant professor and Dy Head of Department at National University of Sciences and Technology (NUST), Islamabad, Pakistan. He received his BEng Industrial Electronics degree from NED University, Pakistan, in 2004. He is MSc Electrical Engineering degree holder from Dalarna University Sweden and National University of Singapore in controls and automation. He received $\mathrm{PhD}$ Degree from University of Southampton, UK. His research interests include digital control design and smart grid technologies. $\mathrm{He}$ is author of several IEEE publications. 